\title{
Anabases
}

ANABASES Traditions et réceptions de l'Antiquité

$4 \mid 2006$

Varia

\section{Un Reinach au service du Daremberg : Théodore Reinach ou la discrète implication d'un helléniste de renom}

Sébastien Delmas

\section{OpenEdition}

Journals

Édition électronique

URL : http://journals.openedition.org/anabases/2998

DOI : $10.4000 /$ anabases.2998

ISSN : 2256-9421

Éditeur

E.R.A.S.M.E.

Édition imprimée

Date de publication : 1 octobre 2006

Pagination : 189-195

ISSN : 1774-4296

Référence électronique

Sébastien Delmas, « Un Reinach au service du Daremberg : Théodore Reinach ou la discrète

implication d'un helléniste de renom », Anabases [En ligne], 4 | 2006, mis en ligne le 01 janvier 2012, consulté le 20 octobre 2019. URL : http://journals.openedition.org/anabases/2998 ; DOI : 10.4000/ anabases. 2998 
Anabases 4 (2006), p. 189-195

\section{Un Reinach au service du Daremberg : Théodore Reinach ou la discrète implication d'un helléniste de renom}

SÉBASTIEN DELMAS

Sur les diZAines D’AUTEURS ayant contribué au Daremberg, Saglio et Pottier combien sont-ils ceux dont les noms résonnent, familiers, aux antiquisants de notre siècle ? Une poignée, tout au plus. Aussi au nombre de ces privilégiés, refusant au Dictionnaire des antiquités grecques et romaines la fonction d'ultime conservatoire de leur activité scientifique, s'étonne-t-on de compter plusieurs représentants d'une seule et même lignée : celle des Reinach. Ce nom, qui en son temps suscitait, à discrétion, superlatifs ou critiques acerbes, fut porté par trois contributeurs : deux frères, Théodore et Salomon, ainsi que le neveu de ceux-ci, Adolphe-Joseph. L'évocation de ces monuments d'érudition nécessiterait autant d'études particulières. Seul l'un d'eux, le premier nommé, mobilisera ici notre attention.

Des membres de sa famille, généreusement pourvue en prodiges, Théodore Reinach (1860-1928) ne fut ni le plus réputé ni le plus sulfureux. Aujourd'hui, plus que jamais, l'évocation de son souvenir se limite généralement à la commémoration du rêve architectural qu'il concrétisait, voilà cent ans, sur la Côte-d'Azur ${ }^{1}$. Mais tous n'ont pas oublié la formidable précocité qui fut la sienne, son savoir encyclopédique; et c'est ainsi qu'il figure à raison parmi les éminents historiens du tournant des XIXe et XXe siècles. Partant, la contribution de ce savant au Daremberg, loin d'être le fait marquant de sa vie intellectuelle est, à l'inverse, une minuscule ligne reversée à son épais dossier bibliographique. Tout l'intérêt consiste donc à analyser la manière dont ce collaborateur " peu ordinaire " concourut à l'entreprise initiée par Charles Daremberg : à quel moment de sa carrière, selon quelles modalités et avec quel degré d'implication ?

1 Le rêve architectural en question n'est autre que la fameuse villa Kérylos, aujourd'hui propriété de l'Institut. 
Arrêtons-nous d'abord aux questions de méthodologie. Théodore Reinach a beau être une importante figure historiographique, se renseigner sur lui près de quatre-vingt ans après sa disparition ne va pas sans difficultés, spécifiquement pour une recherche n'étant approfondie jusqu'à la consultation d'archives. Les sources le concernant, de prime abord, sont à la fois nombreuses et variées : pas moins de trois notices nécrologiques ${ }^{2}$, des entrées dans les dictionnaires biographiques spécialisés (parfois même aussi généraux que le Amalvi ${ }^{3}$ ). D'autres documents encore, comme cette brochure des années 50 au parfum hagiographique ${ }^{4}$; sans compter les informations fournies par les ouvrages traitant des Juifs sous la Troisième République ${ }^{5}$, ou ceux consacrés plus directement à ses frères ${ }^{6}$. Malgré cela, Théodore est de loin le « Reinach première génération " le plus négligé, au profit de ses aînés, Joseph, le politicien, et Salomon, l'archéologue : l'absence de publications à sa seule attention suffit à en attester. Surtout, cette relative notoriété n'empêche pas d'être fort mal renseigné sur sa participation au Daremberg, dont-il est rarement fait état.

Le parcours de Théodore Reinach n'est pas à proprement parler l'objet de cette étude mais l'éluder serait inconcevable. Voici donc, résumée en quelques lignes, une vie qui en mériterait bien davantage. Des origines de ce savant on retiendra la grande aisance d'une famille juive venue de Suisse et l'éducation dans le culte de la République naissante. Benjamin de sa fratrie, Théodore Reinach connut un parcours scolaire brillantissime, de ceux que la superposition du talent à l'émulation familiale autorise quelquefois. Doté très jeune d'un double doctorat, en droit puis en lettre, c'est finalement vers l'histoire ancienne et, plus spécifiquement, celle du monde hellénique oriental qu'il se tourna finalement. Cumulant les responsabilités, enseignant dans les principales institutions parisiennes, il devint en 1909 membre de l'Académie des Inscriptions et Belles-lettres, alors même qu'il était, depuis trois ans déjà et pour six ans encore, député. "Maître des sciences auxiliaires ", Théodore Reinach fut un grand spécialiste de numismatique, de papyrologie ou de musicologie. Joignez à cela la grande attention portée au droit grec, à l'histoire du judaïsme antique, et se parfait le portrait d'un " touche-à-tout " de génie.

2 R. CAGNAT, “ Notice sur la vie et les travaux de Théodore Reinach ”, CRAI, 1931, p. 374393 ; P. JАмОт, Gazette des beaux arts, nov. 1928, 8 p. ; S. DE RICCI, “Théodore Reinach ”, REJ 172, oct-déc. 1928, p. 113-130. C. Amalvi, Dictionnaire biographique des historiens français et francophones, Paris, La Boutique de l'Histoire, 2004, p. 268-269. C. PICARD, "Théodore Reinach ”, in C. Picard (sous la dir. de), À la mémoire des Reinach, Paris, Société d'éditions françaises et internationales, 1950, p. 41-46. P. Birnbaum, Les fous de la République, histoire politique des Juifs d'État de Gambetta à Vichy, Paris, Fayard, 1992.

6 Hervé Duchêne, en particulier, a largement commenté la vie et l'œuvre de Salomon Reinach. 
Ces quelques rappels biographiques n'ont pas d'utilité qu'informative. Ils deviennent même fondamentaux à qui entend déterminer les raisons d'une participation au Dictionnaire des antiquités grecques et romaines. On peut dire, sans exagérer, que Théodore Reinach possédait le profil du candidat idéal ! Il avait pour lui d'être français, de conduire sa carrière à Paris et d'avoir mené de brillantes études. Pour ne rien altérer, il était également l'archétype de l'homme de réseau, qu'il s'agisse des siens propres (juristes, professeurs, élites juives) ou de ceux du clan Reinach (personnel politique, grands financiers). La connexion avec le Daremberg et Saglio put notamment se faire par le biais d'Edmond Pottier, grand ami de Salomon depuis les fouilles de Myrina qu'ils co-dirigèrent. Dernier élément d'explication, les facultés multiples de ce savant d'exception, particulièrement utiles à une entreprise amenée à s'aventurer dans des domaines largement dépourvus de spécialistes. Sans compter que, loin d'œuvrer constamment en solitaire, celui-ci était rompu à l'établissement de corpus en collaboration ${ }^{7}$.

Reinach avait donc toute raison de participer au Dictionnaire, et il y participa en effet. Dès lors que représenta cette collaboration dans sa vie de chercheur ? En consultant la liste des auteurs associés à l'entreprise, on constate qu'il prit part au projet à compter du deuxième tome, paru en 1892, pour ne plus cesser d'y contribuer, jusqu'à la publication du cinquième et dernier opus en 1919. La sollicitation courut donc depuis les environs de sa trentième année jusqu'au-delà de la cinquantaine. Le benjamin des Reinach fit partie de cette seconde génération de contributeurs qui intégrèrent, au sortir de leurs études, l'entreprise en marche, pour l'accompagner à son terme. Au niveau professionnel, comme attendu, les vingt années durant lesquelles Reinach concourut au Daremberg, Saglio et Pottier correspondent au sommet de son implication dans le monde savant. Il n'en va pas différemment de sa production scientifique. De fait la bibliographie considérable qu'il constitua, peu à peu, a beau déborder de ces limites temporelles, c'est en leur sein que l'on retrouve les titres phares de sa production. Les évoquer entièrement serait par trop fastidieux ; citons, pêle-mêle, cet article de 1893, demeuré célèbre, où il parvint à déchiffrer une notation musicale fortuitement découverte ${ }^{8}$, L'histoire par les monnaies (1902), ou encore l'édition des ouvres complètes de Flavius Josèphe (à partir de 1900).

Passées ces premières données, générales quoique indispensables, la participation de Reinach au projet Daremberg et Saglio appelle une analyse attentive de la structure de sa collaboration. Durant le laps de temps où il fut impliqué dans l'entreprise, l'érudit de Saint-Germain-en-Laye prit à sa charge vingt-deux notices, cinq par tome en moyenne. En voici le détail :

$7 \quad$ Citons pour exemple le Recueil général des monnaies grecques d'Asie Mineure (Paris, 19041912), fruit de sa collaboration avec Ernest Babelon.

8 T. REINACH, “La musique des hymnes de Delphes ”, $B C H 17$ (1893), p. 569-616. 


\begin{tabular}{|c|c|c|c|c|c|}
\hline Tome & Année & Lettres & $\begin{array}{l}\text { Nombre } \\
\text { de notices }\end{array}$ & $\begin{array}{l}\text { Nombre } \\
\text { de pages }\end{array}$ & Détail des articles \\
\hline $\mathrm{I}, 1$ & 1877 & $A-B$ & 0 & & \\
\hline I, 2 & 1887 & $\mathrm{C}$ & 0 & & \\
\hline \multirow[t]{12}{*}{ II, 1} & 1892 & D-E & 12 & 1 & Eleuthéra agora \\
\hline & & & & 1 & Eleuthérôn phtora \\
\hline & & & & 1 & Eleuthéroprasiou dikè \\
\hline & & & & 2 & Embatèrion \\
\hline & & & & 1 & Emmènoi dikai \\
\hline & & & & 1 & Emporikai dikai \\
\hline & & & & 1 & Emporikos nomos \\
\hline & & & & 1 & Enoikiou dikè \\
\hline & & & & 3 & Eranos \\
\hline & & & & 1 & Erèmos dikè \\
\hline & & & & 1 & Eupatoristai \\
\hline & & & & 1 & Exagôgès dikè \\
\hline II, 2 & 1896 & F-G & 0 & & \\
\hline \multirow[t]{4}{*}{ III, 1} & 1900 & $\mathrm{H}-\mathrm{K}$ & 4 & 2 & Hymnodus \\
\hline & & & & 7 & Hymnus \\
\hline & & & & 3 & Hyporchèma \\
\hline & & & & 16 & Judaei \\
\hline \multirow[t]{2}{*}{ III, 2} & 1904 & L-M & 2 & 14 & Lyra \\
\hline & & & & 16 & Musica \\
\hline IV, 1 & 1907 & $\mathrm{~N}-\mathrm{Q}$ & 1 & 7 & Paean \\
\hline \multirow[t]{2}{*}{ IV, 2} & 1911 & R-S & 2 & 1 & Symphonia \\
\hline & & & & 4 & Syrinx \\
\hline V & 1919 & $\mathrm{~T}-\mathrm{Z}$ & 1 & 22 & Tibia \\
\hline
\end{tabular}

Fig. 1 : Théodore Reinach et le DAGR

La première constatation réside dans la répartition inégale des entrées dont il assuma la rédaction, incluses pour moitié dans le volume consacré aux lettres $\mathrm{D}$ et $\mathrm{E}$. Mais ce déséquilibre n'est qu’apparent. En deux notices et trente pages, Reinach prit une part proportionnellement plus importante à la constitution du tome III, 2 qu'il ne le fit en douze articles, excédant rarement quelques lignes, pour celle du tome II, 1. 
Autre observation : deux thèmes ressortent nettement d'un rapide parcours de l'intitulé des articles ; le droit grec, omniprésent à la lettre $\mathrm{E}$ ( Erèmos dikè », " Emmènoi »...), et la musicologie («Tibia », « Hymnus »...). Ces aspects réduisent d'ailleurs à la portion congrue les articles relevant d'un sujet différent. Ils ne sont guère que deux, longs toutefois : «Eranos » et « Judaei ».

En prêtant attention à la date des articles, la bipartition thématique s'éclaire d'un jour nouveau. S'il n'était la notice "Embatèrion ", les déclinaisons musicologiques feraient la matière exclusive des interventions postérieures à 1892. Dans un même temps, les écrits relatifs au droit grec se concentrent sur cette seule année 1892. Or on ne peut qu'être frappé de la cohérence liant l'activité du chercheur Théodore Reinach à celle du collaborateur du Dictionnaire. En 1891-1893 l'historien/juriste suppléait Haussoulier et Dureste pour mener à terme le Recueil des inscriptions juridiques grecques, dix années plus tard il proposait une édition du Péri mousikès de Plutarque...

L'agencement des articles juridiques est aisé à comprendre. Courts, car uniquement composés de brèves définitions, ils auraient certainement pu échoir à n'importe quel helléniste au fait de ces questions et présentent assez peu d'intérêt. Ce sont là sans doute des articles de commande destinés à soulager le labeur des collaborateurs les plus actifs. Peut-être permirent-ils aussi de tester le travail du jeune antiquisant qu'était encore Reinach. Quant aux entrées "Judaei " et "Eranos ", sans lendemains, elles doivent s'expliquer par des monographies sur ces sujets ${ }^{9}$. Le principal intérêt est à chercher dans la structure des efforts musicologiques de Reinach. Les notices, singulièrement plus longues, trahissent une attention toute différente. Au reste l'une d'elles n'est autre que l'entrée « Musica » du dictionnaire, preuve du crédit accordé à l'érudit sur ces questions. L'organisation se veut ramifiée. À partir d'un article clef, notre auteur se vit confier la rédaction d'un certain nombre de liens, relatifs aussi bien aux instruments de musique - « Lyra » - qu'aux contextes justifiant leur emploi - « Paean ».

En résumé, il semble que Reinach ait avant tout apporté au Dictionnaire des antiquités son expertise dans les domaines qu'il maîtrisait mieux que quiconque, musicologie en tête. Qu'ils ne lui eussent été proposés ou qu'il se fût refusé à les traiter, les thèmes de civilisation plus classiques revinrent, le plus souvent, à des contributeurs de moindre renom. C'est ainsi que Théodore Reinach, une fois n'est pas coutume, peine à se démarquer des savants constituant la table des auteurs. D'où cette question légitime : peut-on parler dans son cas d'auteur-type, de collaborateur lambda?

Son âge, sa bonne intégration dans les réseaux plaident en ce sens ; restent ses articles. Par leur nombre, ceux-ci le placent dans une catégorie, certes largement représentée, mais nettement mise en minorité par rapport aux auteurs " d'un jour ». Une relative continuité peut, en outre, se percevoir dans l'implication du savant, continuité inaccessible à nombre d'auteurs, même largement impliqués, dont l'activité se circons-

9 Ce qui est vrai de « Judaei » l'est sans doute aussi d' «Eranos». Le doute provient de l'absence d'études sur le sujet référencées dans les bibliographies non exhaustives parcourues. 
crit à un ou deux volumes. Reinach, à l'évidence, pourrait s'insérer dans une catégorie aux contours incertains mais qui ne semble pas être qu'une vue de l'esprit : celle des grands noms du Daremberg. Le concours de ces savants dominant la scène scientifique de leur époque parât avoir été réclamé et obtenu, mais, pour une raison indéterminée, l'implication d'une partie d'entre eux, bien qu' inscrite dans la durée, est demeurée légèrement en retrait. Ce qui est vrai pour Théodore Reinach se vérifie également pour un Salomon Reinach, ou un Paul Foucart. Était-ce lié à une activité déjà trop riche ? Fautil y voir la volonté des concepteurs de ne pas laisser d'empreintes individuelles trop fortes sur leur entreprise ? Ou bien tout autre chose ? La question reste en suspens.

Pour maintenir une certaine logique dans la progression, un zoom sur les entrées semble, après ce regard en surface, tout désigné. En vertu des disparités existantes, les articles consacrés à la musique dans l'Antiquité retiendront plus spécifiquement notre attention.

Dans ses notices, Théodore Reinach reste globalement en retrait. Le ton est plutôt neutre, le on et le nous omniprésents. L'historien laisse à son érudition le soin de le trahir : consacrerait-on, aujourd'hui, 22 pages au seul aulos? Les propos se veulent donc exhaustifs, seulement, ouvrage illustré oblige, ils se voient enrichis d'un appareil iconographique extrêmement riche. Documents figurés, notations musicales, statues, papyrus, monnaies... il ne semble pas qu'une seule classe de documents renseignant sur le rapport des Grecs à la musique ait été mise à l'index. Connaissant l'implication acharnée d'Edmond Saglio dans la compilation de ses sources qui, aujourd'hui encore, font la richesse du Dictionnaire, on ne sait dans qu'elle mesure la réunion du matériel historique est imputable à Reinach. Une chose demeure certaine : les procédés déployés pour servir les propos sont remarquables de diversité et de didactisme.

Les articles signés "Th. Reinach " se révèlent largement descriptifs. Cela se comprend aisément de l'évocation d'un instrument; cela se comprend moins de l'entrée " Musica 10 ». Par chance ces longues notices écrites dans un style très personnel, véritable cachet littéraire, garantissent une lecture agréable. Suggérons, néanmoins, que le Reinach intronisé musicologue n'est pas exactement le vulgarisateur réclamé par l'entreprise. Le constat a ceci de surprenant que la vulgarisation érudite constituait alors le cheval de bataille de son propre frère, Salomon. Une explication, et une seule, peut être invoquée : l'extrême complexité des questions abordées. Malgré toute sa bonne volonté, l'historien ne pouvait rendre abordable à des néophytes des thèmes destinés aux « musiciens qui savent un peu de grec " et aux "hellénistes qui savent un peu de musique ", comme il se plairait à l'écrire ${ }^{11}$. À sa décharge aussi, ne manquera-t-on pas de souligner la limpidité des seize pages dévolues au judaïsme.

Il est difficile de se prononcer sur la place accordée dans les articles aux hypothèses personnelles, de savoir dans quelle mesure les " peut-être " et autres " probablement "

10 Exception faite de réflexions conduites sur la place de la musique dans la vie des Grecs.

11 Propos introductifs de sa Musique grecque (Paris, Payot, 1926). 
dissimulent des opinions propres ou de réelles incertitudes. Il est toutefois quelques moments où le scientifique, sorti de sa réserve, fait écho aux débats historiographiques de son temps. Ainsi ces quelques phrases concluant l'article «Embatèrion » où l'on surprend Théodore Reinach, oublieux du contexte de ses propos, railler les dires d'un auteur allemand : "Quant à l'opinion qui confond les Elégies de Tyrtée avec ses Embatêria, c'est une vieille erreur cent fois réfutée qu'on s'étonne de rencontrer encore dans des ouvrages récents ${ }^{12}$. " Constatons enfin la mise à profit des dernières avancées scientifiques, en particulier celles imputables à notre brillant musicologue. Le péan delphique, interprété par ses soins en 1893, agrémente ainsi le contenu de plusieurs articles.

Chaque notice fait une présentation plus que complète de l'état des connaissances. Lérudition est au centre de tout, l'analyse du sens des pratiques n'intéresse guère ce représentant majeur de la science de son époque. La question de la pertinence, de la résistance au temps des contributions de Reinach se doit donc d'être posée. D'ailleurs, s'il serait mal venu de juger précipitamment cet immense spécialiste de la musique antique, compositeur et exécutant à ses heures, il parait bien difficile, au jour d'aujourd'hui, d'utiliser ses notices autrement que comme autant de vecteurs d'information, aisément accessibles, sur les aspects concrets et factuels de la musique grecque. Les spécialistes contemporains abondent en ce sens, qui ont largement cessé de convoquer ses travaux dans leurs apparats critiques. Et quand, parfois, le nom pionnier de Reinach vient à figurer en commentaire, l'admiration, manifeste, ne le cède en rien à la critique ${ }^{13}$.

L'analyse invite, finalement, à retenir de la collaboration de Théodore Reinach au Dictionnaire des antiquités grecques et romaines l'implication assez ordinaire d'un savant qui ne l'était pas. Inaugurant sa contribution, jeune encore, sans jamais se retirer complètement, celui-ci ne fut, comme tant d'autres, qu'un collaborateur assez régulier plutôt que très actif. Pour " discrète ", cette participation ne s'en fit pas moins en harmonie, avec l'avancement de ses recherches comme avec l'esprit de l'ambitieux projet auquel il participa. Successivement juriste puis musicologue, Théodore Reinach prit soin d'informer sans jamais transiger quant à la transmission de son savoir prodigieux. C'est ainsi sans surprise que l'on observera derrière chaque notice le poids d'un siècle, dans toute la splendeur de ses vertus et de ses limites, tant le personnage qui en assura la rédaction et ceux qui la réclamèrent étaient marqués de son sceau.

\begin{tabular}{l} 
SÉBASTIEN DELMAS \\
\hline 2 rue Cambard - App.D 108 \\
31200 - Toulouse \\
sebastien.delmas1@laposte.net
\end{tabular}

12 s.v. Embatèrion, DAGR, t. II, 1, 1892, p. 596.

13 A. Belis, "Esthétique musicale du péan" in P. Brulé. - C. Vendries (dir.), Chanter les dieux. Musique et religion dans l'Antiquité grecque et romaine, 2001, Rennes, Presses Universitaires de Rennes, p. 109. 